\title{
Caractérisation physico-chimique et évaluation du risque d'eutrophisation du lac Nokoué (Bénin)
}

\author{
Daouda MAMA ${ }^{1,2 *}$, Martin AINA ${ }^{2}$, Abdoukarim ALASSANE ${ }^{2}$, \\ Ousmane Touré BOUKARI ${ }^{2}$, Waris CHOUTI ${ }^{2}$, Véronique DELUCHAT ${ }^{1}$, \\ James BOWEN ${ }^{3}$, Abel AFOUDA ${ }^{2}$ et Michel BAUDU ${ }^{1}$ \\ ${ }^{1}$ Laboratoire : Groupe de Recherche Eau Sol Environnement- EA 4330, 123, Avenue Albert Thomas, 87060 \\ Limoges Cedex (France). \\ ${ }^{2}$ Laboratoire d'Hydrologie Appliquée (LHA), Faculté des Sciences et Techniques, Université d'Abomey- \\ Calavi 01 BP 526 Cotonou (Bénin). \\ ${ }^{3}$ Department t. of Civil Engineering, University of North Carolina at Charlotte, Charlotte NC 28223 (USA). \\ *Auteur correspondant, Email : mkdaouda@yahoo.fr; Tél : 0022996638124
}

\section{RESUME}

L' étude, menée sur deux (2) ans (2006 et 2007) , a permis de faire le bilan hydrologique du lac Nokoué et de connaitre la qualité physico-chimique des eaux du lac en vue d'offrir les éléments de base pour une modélisation écologique future du système. Les données historiques observées au niveau des stations de Bonou et de Sô - Ava, de même que des mesures ponctuelles des débits à l'ADCP des tributaires à leurs entrées dans le lac, ont été exploitées pour réaliser ce bilan hydrologique. Les saisons (saison sèche et période de hautes eaux) ont été prises en compte dans cette évaluation. Les résultats du bilan hydrologique montrent que le débit moyen d'eau douce reçu par le lac en période de hautes eaux (Août à Octobre) est de $216 \mathrm{~m}^{3} / \mathrm{s}$ essentiellement apporté par le fleuve Ouémé, le plus long fleuve du Bénin qui traverse le pays du nord au sud. Cette valeur tombe à $49 \mathrm{~m}^{3} / \mathrm{s}$ en saison sèche avec $86 \%$ du débit apporté par la Sô. En saison sèche, la Sô à Ganvié a le taux le plus important de charge organique (53\% de DBO). Pour le phosphore en saison sèche le plus fort taux est obtenu au niveau du fleuve Ouémé à Totchè (42\%). En période de hautes eaux (Août à Octobre) il est observé un effet de dilution des apports. En terme de charges nutritives apportées par les systèmes d'assainissement, les résultats obtenus sont pour Cotonou et Calavi $360 \mathrm{~kg} / \mathrm{j}-\mathrm{P}$ et $840 \mathrm{~kg} / \mathrm{j}-\mathrm{N}$ et pour Ganvié $150 \mathrm{~kg} / \mathrm{j}-\mathrm{P}$ et 700 $\mathrm{kg} / \mathrm{j}-\mathrm{N}$; les apports des «Acadjas » donnent 2,3 - 2,9 kg/j P et $29-36 \mathrm{~kg} / \mathrm{j} \mathrm{N}$. Les principaux résultats obtenus pour la caractérisation physico - chimique se présentent comme suit :- une intrusion saline est très remarquable en saison sèche, ainsi à Ganvié on passe de $0 \mathrm{~g} / \mathrm{L}$ en période de hautes eaux à $2,5 \mathrm{~g} / \mathrm{L}$ en saison sèche ; le $\mathrm{pH}$ moyen varie ente 6,6 et 7,5 pour les eaux du fond du lac et entre 6,9 et 7,8 pour les eaux de surface du lac. Le $\mathrm{pH}$ varie très peu entre le fond du lac et la surface du lac; l'évaluation du risque d'eutrophisation à partir de l'outil de diagnostic d'Ifremer, classe la quasi-totalité des eaux dans la zone rouge (i.e mauvaise). Les valeurs des nutriments azotés et phosphorés sont largement supérieures aux valeurs limites indiquées par le système de classification de l'Organisation de Coopération et Développement Economique (OCDE). Les eaux du lac sont eutrophes. Cette eutrophisation se manifeste par la prolifération des jacinthes d'eau. On note une évolution saisonnière du système. Du point de vue des facteurs de contrôle des jacinthes d'eau, il ressort que les nutriments contrôlent le développement des jacinthes lorsque la salinité n'inhibe plus la croissance des jacinthes, c'est-à-dire de juillet à janvier.

(C) 2011 International Formulae Group. All rights reserved.

Mots clés : Bilan hydrologique, lac Nokoué, charges polluantes, eutrophisation, jacinthe d'eau, intrusion saline. 


\section{INTRODUCTION}

Les grands lacs estuaires comme le lac Nokoué au Bénin (Afrique de l'Ouest) subissent une forte pression démographique liée à leur situation en zones humides. Dans les lacs, les apports massifs de nutriments liés aux multiples activités anthropiques ont provoqué très souvent l'eutrophisation, caractérisée par la prolifération de la jacinthe d'eau (Ogutu - Ohwayo et al., 1997 ; Mama, 2010).

La prolifération des macrophytes est la principale problématique associée à l'eutrophisation en zone tropicale et principalement en Afrique, à l'opposé des zones tempérées où les cyanobactéries occupent une place de choix (Hill \& Coetzee, 2008).

En zone côtière, les lagunes peuvent avoir des usages multiples (activités récréatives, pisciculture, conchyliculture, thermalisme), ce qui accroit la pression anthropique sur ces systèmes. La présence du lac Nokoué qui abrite les villages atypiques des pêcheurs sur pilotis appelé «villages lacustres » comme Ganvié et le flux touristique augmentent le niveau des pressions anthropiques.

Le risque d'eutrophisation des plans d'eau en zone estuaire peut être évalué à partir de l'outil de diagnostic d'Ifremer en prenant en compte les caractéristiques des sédiments et de la colonne d'eau (Trinquier, 2009).

La croissance de la jacinthe d'eau atteint souvent des proportions d'infestation alarmante imputable à l'eutrophisation surtout dans les lacs peu profonds dont les bassins versants sont soumis à de multiples activités humaines (Feuchtmayr et al., 2009). La croissance des jacinthes d'eau est souvent rythmée par les variations saisonnières et les différents facteurs environnementaux (Hadj et al., 2008). En zone estuaire, la salinité, même à de faibles niveaux, a un effet préjudiciable sur la croissance de la jacinthe d'eau. (Mama, 2010). La salinité, la température et les nutriments sont considérés comme des facteurs qui gouvernent la croissance des jacinthes d'eau en zone estuaire (Wilson et al., 2001).

L'objectif de ce travail est de contribuer à combler le déficit de connaissance $\mathrm{du}$ système lacustre (hydrodynamique et qualité), d'évaluer les différentes charges auxquelles il est soumis avec une bonne indication des variabilités saisonnières.

\section{MATERIEL ET METHODES Site d'étude}

Le lac occupe une superficie d'environ $150 \mathrm{~km}^{2}$ en période d'étiage. La profondeur moyenne du lac est de $1,5 \mathrm{~m}$. La bathymétrie du lac Nokoué montre des valeurs caractéristiques des lacs peu profonds. La profondeur est inférieure à $1 \mathrm{~m}$ sur $20 \%$ de la surface du plan d'eau. La zone la plus profonde $(8 \mathrm{~m})$ se situe au niveau de l'entrée du chenal de Cotonou.

Une diminution de l'ordre de $0,6 \mathrm{~m}$ de la profondeur maximale a été observée sur environ 20 ans (1986 à 2006) soit 0,03 m/an environ. La forte utilisation des pièges à poissons «Acadjas» et les apports sédimentaires liés à l'hydrodynamique du lac constituent les principales causes de cette tendance au comblement du lac.

Les principaux tributaires du lac sont:

- l'Ouémé, de bassin versant 46500 $\mathrm{km}^{2}$ et de longueur $523 \mathrm{~km}$, traverse le pays du Nord au Sud. Il est, en termes d'apport d'eau douce, largement sous l'influence de la pluviométrie de son bassin supérieur (Ouémé supérieur);

- la Sô, de bassin versant $1000 \mathrm{~km}^{2}$ et de longueur $70 \mathrm{~km}$ est connectée au fleuve Ouémé en haute eaux et entretien un bon niveau de débit en saison sèche ;

- le chenal de Cotonou de longueur 4,5 $\mathrm{km}$, de largeur $300 \mathrm{~m}$ et une profondeur variant entre 5 et $10 \mathrm{~m}$. Il est le tributaire d'eau de mer du lac Nokoué.

\section{Echantillonnage}

Le choix des points d'échantillonnage a été effectué de façon raisonnée afin d'avoir des informations plus précises sur le secteur 
du lac le plus anthropisé. Par ailleurs, il était important de pouvoir réaliser la campagne de suivi, effectuée en barque, en une journée. Ainsi 7 points de prélèvement ont été définis.

- Deux points S1 et S2 encadrent le village lacustre de Ganvié, mais par la suite, seul le point $\mathrm{S} 1$ a été considéré. L'objectif de ce prélèvement est d'avoir une estimation de l'impact du village lacustre de Ganvié sur le lac.

- Le point $\mathrm{S} 3$, situé à Sô - Ava (station de suivi des hauteurs d'eau) renseigne sur la qualité de l'eau de la rivière Sô.

- Le point S4 est situé à l'entrée du village Sôtchanhoué en partant de la branche de la seconde branche de la Sô. Il permet d'apprécier l'influence des eaux du fleuve Ouémé (marécages de la zone deltaïque).

- Le point S5, situé pratiquement au centre du lac, permet d'apprécier la dynamique de mélange des eaux du lac. Les jacinthes sont assez peu présentes dans ce secteur, de même que dans la partie Est du lac.

- Le point S6, situé à l'entrée du chenal de Cotonou, permet de suivre l'effet conjugué des collecteurs de Cotonou, des apports du côté Ouest du lac et de l'océan.

- Le point $\mathrm{S} 7$, correspond à une zone d'arrivée d'eaux usées d'une partie de la ville de Calavi.

\section{Mesure des débits par ADCP}

La détermination des débits d'entrée au niveau des tributaires en saison sèche et en saison de hautes eaux, doit permettre de combler le déficit d'information au niveau des tributaires. Les mesures de débit et de profondeur ont été effectuées à l'aide d'un appareil à effet Doppler de marque RD Instruments. L'appareil est fixé sur une planche attachée à la barque et plongée dans l'eau. Il est relié à un ordinateur doté du logiciel (Win River Application) qui calcule le débit par intégration du champ de vitesse dans la section.

\section{Evaluation des charges polluantes des principaux tributaires (Fleuve Ouémé- rivière $\mathrm{Sô}$ ) \\ Les données historiques des débits} recueillies aux niveau de certaines stations (Bonou et S)o-Ava) ont été complétées par les mesures ponctuelles des débits effectuées à l'ADCP. Les moyennes des concentrations des polluants suivis entre 2006 et 2007 (Mama, 2010) ont servi de base au calcul des charges polluantes. Dans le cas particulier de la Sô, les deux entrées dans le lac (par Ganvié et par Sotchanhoué) ont été considérées avec une répartition ( $2 / 3$ et $1 / 3$ respectivement) du débit mesuré à Sô - Ava, sur la base des rapports des sections des lits des 2 entrées de la Sô dans le lac.

\section{Méthode d'analyse}

Pour cerner l'état de la qualité de cette eau, nous nous sommes intéressés aux paramètres associés à l'eutrophisation (Ryding \& Rast, 1994) et à la dynamique de croissance des jacinthes d'eau (Wilson et al., 2005). Ces paramètres sont la salinité, le $\mathrm{pH}$, la concentration en oxygène dissous, les composés azotés ( $\mathrm{NO}_{3}{ }^{-}$et NTK), les composés phosphorés (phosphate et phosphore total), la matière organique $\left(\mathrm{DBO}_{5}\right)$.

Le $\mathrm{pH}$, la salinité et la concentration en oxygène dissous sont également mesurés in situ à l'aide d'un capteur multi paramètres PC HORIBA WATER QUALITY CHECKER U- 10 .

La DBO a été par un DBOmètre de la marque OxiTop. L'azote Kjeldahl a été déterminé selon la norme AFNOR NFT 90 110.

La concentration en nitrate été déterminée à l'aide de Kits MERCK et HACH. Les mesures spectrométriques ont été effectuées soit à l'aide du spectrophotomètre MERCK Spectroquant NOVA ou du spectrophotomètre HACH LANGE DR 2800.

Les orthophosphates et le phosphore total sont dosés par spectrométrie en détectant 
le complexe phosphomolybdique de couleur bleu, selon la norme NFT $90-023$.

Le système lacustre a ainsi été caractérisé au plan quantitatif par la mesure des débits et l'estimation des charges. Le suivi des paramètres de qualité a été effectué sur 2 ans (2006 - 2007).

Analyse des résultats à partir de l'outil de diagnostic de l'eutrophisation de Ifremer

La caractérisation de l'état trophique a été réalisée sur les sédiments et l'eau à l'aide de la grille de diagnostic d'Ifremer élaborée en 2000. Pour les sédiments, la grille prend en compte la matière organique, l'azote total et le phosphore total. Les paramètres considérés pour la colonne d'eau sont : Phosphate; nitrate; ion ammonium ; chlorophylle a ; azote total et phosphore total en prenant en compte 2 niveau (la surface et le fond).

Les moyennes des valeurs observées de Février à Novembre 2007, ont été utilisées pour classer chaque station selon les paramètres de la grille d'évaluation d'Ifremer aussi bien pour les prélèvements de surface que pour les prélèvements de fond du lac. Les valeurs moyennes ainsi obtenues sont comparées à celles retenues par la grille pour estimer le risque d'eutrophisation du lac. Dans le cas présent, outre la colonne d'eau, les sédiments ont été priss en compte pour quatre (4) stations.

\section{RESULTATS}

Bilan hydrologique

Les résultats des estimations des débits sont donnés par le Tableau 1 ci-dessous.

Répartition des charges organiques et nutritives (azote et phosphore)

De la Figure 2, il apparaît qu'en saison sèche, les charges en DBO et en azote sont plus importantes au niveau de Ganvié (58\%) et Sôtchanhoué (29\%). Pour cette saison, les apports du fleuve Ouémé sont les plus faibles. En ce qui concerne la matière phosphorée, la charge apportée par l'Ouémé à Totchè (42\%) est la plus importante. En période de hautes eaux, on assiste à un effet de dilution importante surtout pour la charge organique dont les apports semblent dépendre surtout des villages lacustres et des eaux usées en provenance des quartiers environnants. Par contre, les apports des matières nutritives et surtout le phosphore semblent provenir des eaux drainées à partir des bassins versants.

Charges apportées par la décomposition des « acadjas » et jacinthe d'eau dans le lac Nokoué

Le Tableau 2 ci-dessous donne les estimations des charges liées à la décomposition des acadjas.

\section{Suivi de la qualité du système \\ Paramètres physico-chimiques - $\quad$ Salinité}

La Figure 3 montre que les eaux du fleuve Ouémé influencent considérablement la dynamique du lac Nokoué. En effet, l'évolution comparée du débit avec la salinité montre que lorsque le débit du fleuve à Bonou est maximum d'août à octobre $\left(800 \mathrm{~m}^{3} / \mathrm{s}\right)$, la salinité baisse considérablement. Inversement, en saison sèche (étiage) des valeurs maximales de salinité sont atteintes alors que le débit du fleuve avoisine $2 \mathrm{~m}^{3} / \mathrm{s}$ à la première station hydrologique (Bonou) en amont du lac.

\section{- $\underline{p H}$}

De la Figure 4, on note une assez faible variabilité de ce paramètre $(<1,7$ unités $\mathrm{pH})$ au sein du lac Nokoué. De même, ces résultats montrent qu'il y a très peu de variation du $\mathrm{pH}$ en passant de la surface au fond du lac pour la majorité des stations suivies, cependant les stations S3 et S5 présentent des variations relativement plus importantes. Ce résultat pourrait s'expliquer par le fait que ce sont les zones dans lesquelles l'activité photosynthétique du phytoplancton est la plus élevée, les mesures étant faites en période diurnes, l'augmentation du pH dans la zone de surface pourrait être reliée à cette observation.

\section{- Oxygène dissous}

Les plus faibles concentrations en oxygène dissous se situent au niveau des 
points S1, S2, S4 et S7, c'est -à-dire dans les zones à forte pression anthropique. Les plus fortes valeurs sont observées aux stations S6 et S5 comme dans le cas de la profondeur au disque de Secchi.

De la Figure 5, on note que les plus faibles valeurs de la teneur en oxygène dissous sont relevées au mois de Février, qui correspond à la période de décomposition des macrophytes.

\section{- Matière organique : DBO5}

$\mathrm{La} \mathrm{DBO}_{5}$ présente des valeurs souvent supérieures à $10 \mathrm{mg} / \mathrm{L}$, donc relativement élevées (Tableau 10). Les plus fortes valeurs observées en Février sont sans doute liées à la décomposition des macrophytes dans le lac. Pour certains points, il est probable que des phénomènes d'inhibition (liés à la présence de toxiques), interférent sur la mesure (cas du point S7, pour lequel les apports d'eaux usées urbaines sont majeurs) (Tableau 3).

\section{- $\quad$ Azote total}

Le suivi sur toute l'année de l'azote montre l'influence des facteurs climatiques sur les apports. Les paramètres azotés comme les nitrates, l'ammonium et les nitrites ont été également suivis (Figure 6).

On observe des concentrations plus élevées en azote total de Février à Avril, période chaude $\left(31-33{ }^{\circ} \mathrm{C}\right)$ à Cotonou comparée au mois d'Août (23 à $\left.25^{\circ} \mathrm{C}\right)$. Le mois de février a coïncidé également avec le début de la décomposition des jacinthes mortes sous l'effet de l'accroissement de la salinité au niveau du lac. Cette phase de décomposition favorise l'augmentation de la concentration en azote dans la colonne d'eau. On notera que les plus fortes concentrations sont généralement observées en S7 et S6, qui sont les zones d'apports directs d'eaux usées domestiques. Les premières pluies du mois d'avril, ainsi que les phénomènes d'oxydation de ces formes réduites de l'azote en nitrate et nitrite, peuvent expliquer la diminution de la concentration en azote Kjeldahl observée en Avril. La suite de l'évolution des apports semble dépendre de l'hydrologie du fleuve, qui induit une dilution des apports d'azote que constituent les eaux usées rejetées directement dans le système.

\section{- $\quad$ Ion Nitrate $\left(\mathrm{NO}_{3}^{-}\right)$}

La Figure 7 présente l'évolution de la concentration en nitrate sur les différents points durant l'année 2007. On observe que leur évolution suit de manière très marquée, l'effet de concentration lié à l'évapotranspiration (concentration élevée en saison sèche) et l'effet de dilution pendant les hautes eaux (Mai - Novembre).

Les plus fortes concentrations en nitrates sont observées au point S7, pour lequel il n'avait pas été observé de concentration très élevée en azote ammoniacal malgré la forte anthropisation de cette zone. Ainsi, malgré des concentrations en oxygène dissous souvent faibles dans cette zone, la nitrification semble bien s'y opérer.

\section{- Le phosphore total}

C'est un paramètre important de la fertilisation des plans d'eau, il joue un grand rôle dans la croissance planctonique. Ce nutriment est un indicateur pertinent de l'évaluation du niveau trophique des eaux. Les valeurs de plus de $800 \mu \mathrm{g}$ P/L sont obtenues au niveau des différents points. Les teneurs en phosphore diminuent au niveau de Bonou, puis restent voisines de $0,8 \mathrm{mg} \mathrm{P} / \mathrm{L}$. Dans le lac, la concentration en $\mathrm{P}$ est partout inférieure à $0,8 \mathrm{mg} / \mathrm{L}$, excepté au niveau du point $\mathrm{S} 4$ où une valeur maximale de $1,52 \mathrm{mg} / \mathrm{Len}$ Novmbre 2007. Le rôle du fleuve Ouémé dans l'enrichissement du système en phosphore semble donc majeur (Figure 8).

En tenant compte de la variation de la pluviométrie à Cotonou, il apparaît que les premières pluies apportent du phosphore par lessivage des bassins versants et des abords généralement occupés par des déchets solides ménagers. Un fonctionnement différent concernant l'enrichissement du milieu en azote et phosphore peut être envisagé. Il apparaît que les eaux usées jouent un rôle majeur dans les apports d'azote, alors que le fleuve Ouémé qui draine les eaux du bassin de l'Ouémé supérieur à forte exploitation d'engrais pour les champs de coton apporterait une grande partie du phosphore. Ce résultat est conforme à ceux obtenus plus haut au cours de l'estimation des charges polluantes. 


\section{- Phosphate $\left(\mathrm{PO}_{4}{ }^{3-}\right)$}

Figure 9 montre que les concentrations en phosphates subissent les effets de dilution en saison de hautes eaux et de concentration en saison sèche avec une amplitude moindre comparée à celle des nitrates.

Globalement les nutriments solubles dans la colonne d'eau sont très sensibles aux effets de dilution dus aux apports d'eau douce de Mai à Novembre et à l'évapotranspiration prolongée en saison sèche, de Novembre à Avril.

Les concentrations les plus élevées sont là aussi observées au niveau de la station S7, sous l'influence des apports de la ville de Calavi. Le point S1 (village de Ganvié) présente également des concentrations en phosphate très élevées (toujours supérieures à $100 \mu \mathrm{g} / \mathrm{L})$.

\section{Résultat de l'analyse du risque trophique à partir de l'outil d'Ifremer}

Les résultats d'analyse des sédiments sont présentés dans les Tableaux 4 et 5.

\section{Colonne d'eau}

La caractérisation de l'état trophique le long de la colonne d'eau des stations du lac Nokoué par l'outil de diagnostic de l'eutrophisation fait ressortir les résultats suivants (Tableaux 6 à 11).
Les résultats montrent que tous les points présentent des eaux de très mauvaise qualité selon la grille de diagnostic d'Ifremer.

$\mathrm{Au}$ niveau de cette station, tous les paramètres sont classés dans la gamme «mauvais » sauf le phosphate qui est classé dans la gamme « moyen » (Tableau 7).

$\mathrm{Au}$ niveau de cette stations, 3 paramètres sont classés dans la gamme «mauvais» et 3 dans la gamme «moyen» (Tableau 8).

$\mathrm{Au}$ niveau de cette station, tous les paramètres sont classés dans la gamme « mauvais » sauf le phosphate et l'ammonium qui sont classés dans la gamme «Médiocre» pour la surface et le phosphate dans la gamme «Bon» pour le fond (Tableau 9).

$\mathrm{Au}$ niveau de cette station, la plupart des paramètres sont classés dans la gamme «mauvais » (Tableau 10). Pour cette station, tous les paramètres sont classés dans la gamme «mauvais » sauf le phosphate qui est classé dans la gamme «médiocre» (Tableau 11). Au niveau de la colonne d'eau, toutes les stations sont dans un mauvais état vis-à-vis de leur état trophique. Au niveau des sédiments, les stations S1 (Ganvié), S2 (Sô - Ava) et S7 (Calavi) sont dans un mauvais état mais la station S5 (centre du lac) est par contre classée dans la gamme «Bon ».

Tableau 1 : Débits d'entrée des tributaires Sô et Ouémé dans le lac Nokoué.

\begin{tabular}{lllllll}
\hline Tributaire & Entrée & Saison/ Période & Débit & $\begin{array}{c}\text { DBO5 } \\
(\mathbf{k g} / \mathbf{j})\end{array}$ & MP $(\mathbf{k g} / \mathbf{j})$ & MN $(\mathbf{K g} / \mathbf{j})$ \\
\hline \multirow{2}{*}{$\begin{array}{l}\text { Fleuve } \\
\text { Ouémé }\end{array}$} & Bonou & Saison sèche & $11 \mathrm{~m}^{3} / \mathrm{s}$ & 1900 & 1140 & 142 \\
& & Hautes eaux & $338 \mathrm{~m}^{3} / \mathrm{s}$ & 29203 & 29203 & 1460 \\
\cline { 2 - 6 } & Totchè / lac & Saison sèche & $7 \mathrm{~m}^{3} / \mathrm{s}$ & 907 & 725 & 90 \\
& Nokoué & Hautes eaux & $140 \mathrm{~m}^{3} / \mathrm{s}$ & 9676 & 12096 & 604 \\
\hline Rivière Sô & Sô- Ava & Saison sèche & $36 \mathrm{~m}^{3} / \mathrm{s}$ & 6220 & 2799 & 124 \\
& & Hautes eaux & $204 \mathrm{~m}^{3} / \mathrm{s}$ & 17625 & 12337 & 352 \\
\cline { 2 - 6 } & Ganvié & Saison sèche & $24 \mathrm{~m}^{3} / \mathrm{s}$ & 4147 & 1866 & 82 \\
& & Hautes eaux & $136 \mathrm{~m}^{3} / \mathrm{s}$ & 11750 & 8225 & 235 \\
\cline { 2 - 6 } & Sôtchanhoué & Saison sèche & $12 \mathrm{~m}^{3} / \mathrm{s}$ & 2073 & 933 & 41 \\
& & Hautes eaux & $68 \mathrm{~m}^{3} / \mathrm{s}$ & 5875 & 4112 & 117 \\
\hline \multicolumn{2}{l}{$\begin{array}{l}\text { Total } \text { charge reçue en saison sèche par le lac } \\
\text { Total } \text { charge reçue en hautes eaux par le lac }\end{array}$} & & 7127 & 3524 & 213 \\
\hline
\end{tabular}


Tableau 2: Estimation des charges apportées par jacinthe d'eau et la décomposition des «acadjas » dans le lac Nokoué.

\begin{tabular}{lcc}
\hline Nutriments & $\begin{array}{c}\text { Charge annuelle des } \\
\text { acadjas }\end{array}$ & $\begin{array}{c}\text { Charge annuelle apportée par la jacinthe } \\
\text { d'eau }\end{array}$ \\
\hline Carbone & 2100 à $2650 \mathrm{t}$ & $39 \mathrm{t}$ \\
$\mathrm{DBO}_{5}$ & 5600 à $7067 \mathrm{t}$ & $104 \mathrm{t}$ \\
Phosphore & 0,84 à $1,06 \mathrm{t}$ & $0,48 \mathrm{t}$ \\
Azote & 10,5 à $13,3 \mathrm{t}$ & $4,2 \mathrm{t}$ \\
\hline
\end{tabular}

Tableau 3 : Résultats des mesures de la DBO5 $\left(\mathrm{mg} / \mathrm{L} \mathrm{O}_{2}\right)$ sur le lac Nokoué.

\begin{tabular}{lcccccrc}
\hline Ech. Date & S1 & S2 & S3 & S4 & S5 & S6 & S7 \\
\hline Novembre 2006 & 10 & 10 & 13 & 8 & 8 & - & 13 \\
Décembre 2006 & 16 & 15 & 15 & - & 15 & 3 & 18 \\
Février 2007 & 22 & 31 & 21 & 30 & 32 & 35 & 14
\end{tabular}

S1 (Ganvié côté Nokoué), S2 (Ganvié côté Sô) ; S3 (Sô-Ava); S4 (Sôtchanhoué) ; S5 (centre du lac) et S7 (Embarcadère Calavi).

Tableau 4: Résultats d'analyse liée aux sédiments (a) Ganvié et (b) Sô-Ava.

(a)

(b)

\begin{tabular}{llcclll}
\hline $\begin{array}{l}\text { Station S1: Village } \\
\text { de Ganvie }\end{array}$ & Médiocre & Mauvais & $\begin{array}{l}\text { Station S2 (Sô- } \\
\text { Ava) }\end{array}$ & Médiocre & Mauvais \\
\hline$O$ & $\%$ & $\mathbf{9 , 5}$ & $\mathrm{MO}$ & $\%$ & $\mathbf{9 , 1}$ & \\
$N T$ & & & & & \\
$P T$ & $\mathrm{~kg} D M$ & $\mathrm{NT}$ & $\mathrm{g} / \mathrm{kg}$ & $\mathbf{4 , 8}$ \\
$P T$ & & $\mathrm{PT}$ & $\mathrm{mg} / \mathrm{kg}$ & $\mathbf{1 9 6 5}$ \\
\hline
\end{tabular}

Tableau 5: Résultats d'analyse liée aux sédiments (a) Centre du lac et (b) Calavi.

(a)

\begin{tabular}{llcccll}
\hline $\begin{array}{l}\text { Station S5 (Centre du } \\
\text { lac) }\end{array}$ & Très bon & Bon & Mauvais & $\begin{array}{l}\text { Station S7 } \\
\text { (Calavi) }\end{array}$ & Mauvais \\
\hline MO & $\%$ & $\mathbf{2 , 4}$ & & OM & $\%$ & $\mathbf{1 9}$ \\
$\mathrm{NT}$ & $\mathrm{g} / \mathrm{kg}$ & & $\mathbf{1 , 2}$ & $\mathrm{NT}$ & $\mathrm{g} / \mathrm{kg}$ & $\mathbf{9 , 5}$ \\
$\mathrm{PT}$ & $\mathrm{mg} / \mathrm{kg}$ & & & $\mathrm{PT}$ & $\mathrm{mg} / \mathrm{kg}$ & $\mathbf{1 5 9 1}$ \\
\hline
\end{tabular}

Tableau 6: Résultats d'analyse des mesures d'eau prises (surface et fond) à la station S1 (Ganvié).

\begin{tabular}{|c|c|c|c|c|c|c|}
\hline \multicolumn{2}{|c|}{ Station S1 (Surface) } & \multirow{2}{*}{$\begin{array}{r}\text { Moyen } \\
\mathbf{1 , 4 7}\end{array}$} & \multirow{2}{*}{$\begin{array}{c}\text { Mauvais } \\
\mathrm{PO}_{4}{ }^{3-}\end{array}$} & \multirow{2}{*}{$\begin{array}{l}\text { Station S1 (Fond) } \\
\mu \mathrm{M}\end{array}$} & \multirow{2}{*}{$\begin{array}{l}\text { Moyen } \\
\mathbf{1 , 4 7}\end{array}$} & \multirow[t]{2}{*}{ Mauvais } \\
\hline $\mathrm{PO}_{4}{ }^{3-}$ & $\mu \mathrm{M}$ & & & & & \\
\hline NITRA & $\mu \mathrm{M}$ & & NITRA & $\mu \mathrm{M}$ & & 21,18 \\
\hline AMMO & $\mu \mathrm{M}$ & & AMMO & $\mu \mathrm{M}$ & & 17,22 \\
\hline Chl-a & $\mathrm{Mg} \cdot \mathrm{m}^{-3}$ & & Chl-a & $\mathrm{Mg} \cdot \mathrm{m}^{-3}$ & & 27,61 \\
\hline NT & $\mu \mathrm{M}$ & & NT & $\mu \mathrm{M}$ & & 212,86 \\
\hline PT & $\mu \mathrm{M}$ & & PT & $\mu \mathrm{M}$ & & 31,87 \\
\hline
\end{tabular}


Tableau 7 : Résultats d'analyse des mesures d'eau prises (surface et fond) à la station S3 (Sô- Ava).

\begin{tabular}{llccllll}
\hline \multicolumn{2}{l}{ Station S3 (Surface) } & Moyen & Mauvais & $\begin{array}{l}\text { Station } \\
\text { S3 (Fond) }\end{array}$ & Moyen & Médiocre & Mauvais \\
\hline $\mathrm{PO}_{4}{ }^{3-}$ & $\mu \mathrm{M}$ & $\mathbf{2 , 8 4}$ & $\mathrm{PO}_{4}^{3-}$ & $\mu \mathrm{M}$ & $\mathbf{1 , 4 7}$ & $\mathbf{1 , 8 4}$ & \\
NITRA & $\mu \mathrm{M}$ & & NITRA & $\mu \mathrm{M}$ & & & $\mathbf{2 0 , 2 9}$ \\
$\mathrm{AMMO}$ & $\mu \mathrm{M}$ & & $\mathrm{AMMO}$ & $\mu \mathrm{M}$ & & & $\mathbf{1 5 , 3 9}$ \\
$\mathrm{Chl}-\mathrm{a}$ & $\mathrm{Mg} \cdot \mathrm{m}^{-3}$ & $\mathbf{1 7 , 9 3}$ & $\mathrm{Chl}-\mathrm{a}$ & $\mathrm{Mg} \cdot \mathrm{m}^{-3}$ & & $\mathbf{1 8 , 4 4}$ & \\
$\mathrm{NT}$ & $\mu \mathrm{M}$ & $\mathbf{1 1 1 , 4 3}$ & $\mathrm{NT}$ & $\mu \mathrm{M}$ & $\mathbf{9 2 , 2 9}$ & & \\
$\mathrm{PT}$ & $\mu \mathrm{M}$ & & $\mathrm{PT}$ & $\mu \mathrm{M}$ & & & $\mathbf{2 9 , 5 3}$ \\
\hline
\end{tabular}

NITR=nitrate $; \mathrm{AMMO}=$ Ion ammonium ; NT= Azote total $; \mathrm{PT}=$ Phosphore total $; \mu \mathrm{M}=$ micomolaire.

Tableau 8 : Résultats d'analyse des mesures d'eau prises (surface et fond) à la station S5 (Centre du lac).

\begin{tabular}{lllrllll}
\hline Station S5 (Surface) & Médiocre & Mauvais & $\begin{array}{l}\text { Station } \\
\text { S5 } \\
\text { (Fond) }\end{array}$ & Bon & Médiocre & Mauvais \\
\hline PO $_{4}{ }^{3-}$ & $\mu \mathrm{M}$ & $\mathbf{2 , 1 0}$ & $\mathrm{PO}_{4}{ }^{3-}$ & $\mu \mathrm{M}$ & $\mathbf{0 , 9 2}$ & & \\
NITRA & $\mu \mathrm{M}$ & & $\mathrm{NITRA}$ & $\mu \mathrm{M}$ & & & $\mathbf{1 5 , 3 9}$ \\
$\mathrm{AMMO}$ & $\mu \mathrm{M}$ & $\mathbf{6 , 0 0}$ & $\mathrm{AMMO}$ & $\mu \mathrm{M}$ & $\mathbf{9 , 3 3}$ & \\
$\mathrm{Chl}-\mathrm{a}$ & $\mathrm{Mg} \cdot \mathrm{m}^{-3}$ & & $\mathrm{Chl}-\mathrm{a}$ & $\mathrm{Mg} \cdot \mathrm{m}^{-3}$ & & & $\mathbf{4 6 , 6 4}$ \\
$\mathrm{NT}$ & $\mu \mathrm{M}$ & & $\mathrm{NT}$ & $\mu \mathrm{M}$ & & & $\mathbf{2 3 9 , 5}$ \\
$\mathrm{PT}$ & $\mu \mathrm{M}$ & & $\mathrm{PT}$ & $\mu \mathrm{M}$ & & & $\mathbf{2 5 , 2}$ \\
\hline
\end{tabular}

$\mathrm{NITR}=$ nitrate $; \mathrm{AMMO}=$ Ion ammonium $; \mathrm{NT}=$ Azote total $; \mathrm{PT}=$ Phosphore total $; \mu \mathrm{M}=$ micomolaire.

Tableau 9: Résultats d'analyse des mesures d'eau prises (surface et fond) à la station S6 entrée chenal de Cotonou.

\begin{tabular}{cccccccc}
\hline \multicolumn{2}{c}{ Station S6 (Surface) } & Moyen & Mauvais & $\begin{array}{c}\text { Station } \\
\text { S6 } \\
\text { (Fond) }\end{array}$ & Moyen & Médiocre & Mauvais \\
\hline $\mathrm{PO}_{4}{ }^{3-}$ & $\mu \mathrm{M}$ & $\mathbf{3 , 1 3}$ & $\mathrm{PO}_{4}^{3-}$ & $\mu \mathrm{M}$ & $\mathbf{2 , 7 2}$ & \\
$\mathrm{NITRA}$ & $\mu \mathrm{M}$ & & $\mathrm{NITRA}$ & $\mu \mathrm{M}$ & & $\mathbf{1 6 , 8 9}$ \\
$\mathrm{AMMO}$ & $\mu \mathrm{M}$ & $\mathbf{6 , 6 1}$ & $\mathrm{AMMO}$ & $\mu \mathrm{M}$ & $\mathbf{4 , 7 2}$ & \\
$\mathrm{Chl}-\mathrm{a}$ & $\mathrm{Mg} \cdot \mathrm{m}^{-3}$ & & $\mathrm{Chl}-\mathrm{a}$ & $\mathrm{Mg} \cdot \mathrm{m}^{-3}$ & & $\mathbf{2 2 , 5 3}$ \\
$\mathrm{NT}$ & $\mu \mathrm{M}$ & & $\mathrm{NT}$ & $\mu \mathrm{M}$ & $\mathbf{9 2 , 2 9}$ & \\
& $\mu \mathrm{M}$ & & $\mathrm{PT}$ & $\mu \mathrm{M}$ & & $\mathbf{1 6 , 6 7}$ \\
\hline
\end{tabular}

$\mathrm{NITR}=$ nitrate $; \mathrm{AMMO}=$ Ion ammonium ; NT= Azote total $; \mathrm{PT}=$ Phosphore total $; \mu \mathrm{M}=$ micomolaire. 
Tableau 10: Résultats d'analyse des mesures d'eau prises (surface et fond) à la station S7 (Calavi).

\begin{tabular}{llcclll}
\hline \multicolumn{2}{c}{ Station S7 (Surface) } & Médiocre & Mauvais & $\begin{array}{l}\text { Station S7 } \\
\text { (Fond) }\end{array}$ & Médiocre & Mauvais \\
\hline PO $_{4}{ }^{3-}$ & $\mu \mathrm{M}$ & $\mathbf{3 , 7 5}$ & $\mathrm{PO}_{4}^{3-}$ & $\mu \mathrm{M}$ & $\mathbf{2 , 0 8}$ & \\
NITRA & $\mu \mathrm{M}$ & & NITRA & $\mu \mathrm{M}$ & & $\mathbf{1 6 , 8 1}$ \\
$\mathrm{AMMO}$ & $\mu \mathrm{M}$ & & AMMO & $\mu \mathrm{M}$ & & $\mathbf{6 1 , 6 7}$ \\
Chl-a & $\mathrm{Mg} . \mathrm{m}^{-3}$ & & $\mathrm{Chl}-\mathrm{a}$ & $\mathrm{Mg} \cdot \mathrm{m}^{-3}$ & & $\mathbf{2 9 , 1 0}$ \\
$\mathrm{NT}$ & $\mu \mathrm{M}$ & & $\mathrm{NT}$ & $\mu \mathrm{M}$ & & $\mathbf{1 3 6 , 2 9}$ \\
$\mathrm{PT}$ & $\mu \mathrm{M}$ & & $\mathrm{PT}$ & $\mu \mathrm{M}$ & & $\mathbf{3 9 , 6 7}$ \\
\hline
\end{tabular}

NITR=nitrate ; $\mathrm{AMMO}=$ Ion ammonium ; NT= Azote total ; PT= Phosphore total ; $\mu \mathrm{M}=$ micomolaire.

Tableau 11 : Bilan du diagnostic de l'eutrophisation dans le lac Nokoué.

\begin{tabular}{llcccccc}
\hline Station & S1 & S2 & S3 & S5 & S6 & S7 & $\begin{array}{c}\text { Bilan } \\
\text { Général }\end{array}$ \\
\hline Colonne d'eau & Mauvais & - & Mauvais & Mauvais & Mauvais & Mauvais & Mauvais \\
Sédiments & Mauvais & Mauvais & - & Bon & - & Mauvais & Mauvais \\
\hline
\end{tabular}

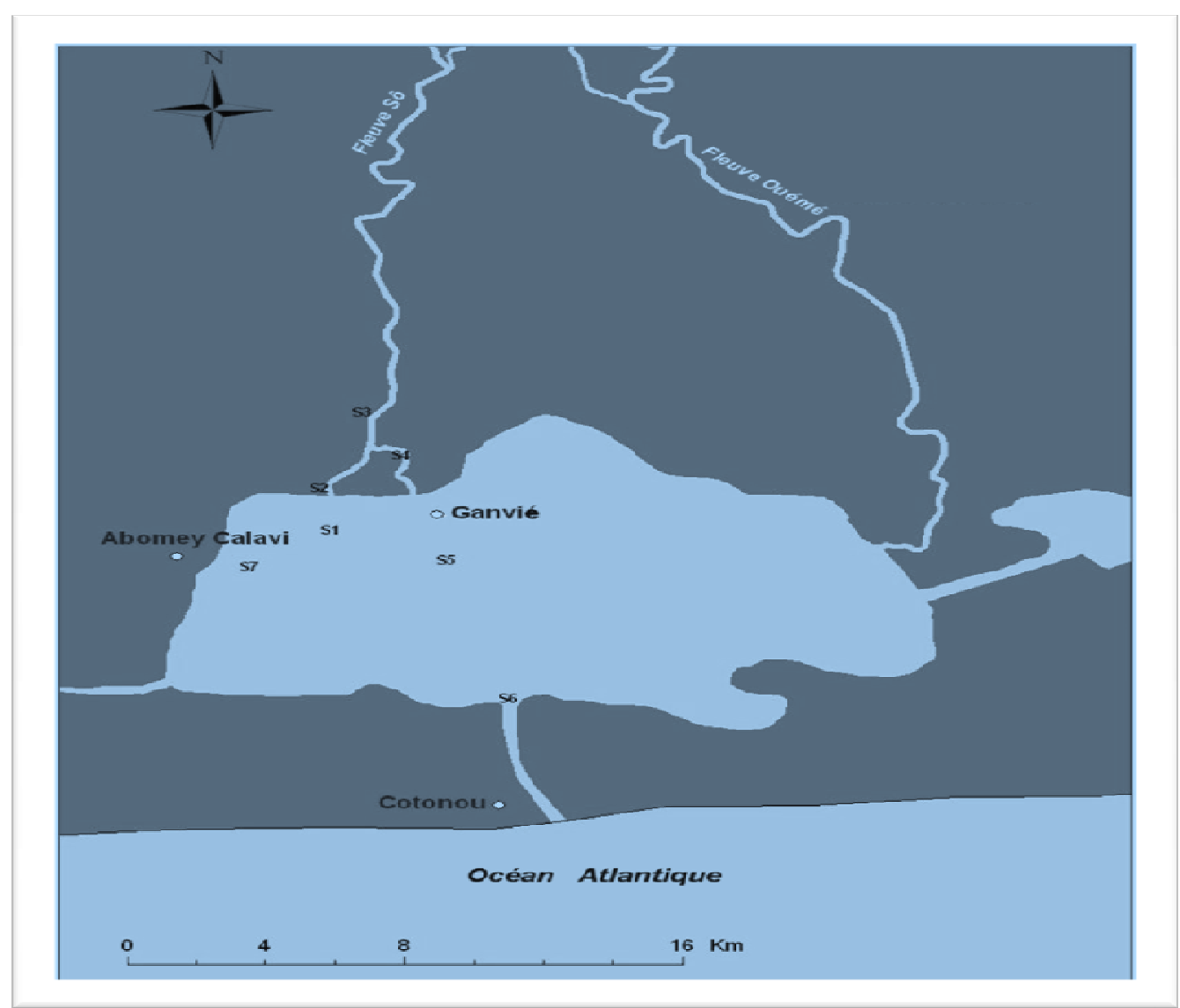

Figure 1: Positionnement des points de mesure sur le lac Nokoué.

S1 (Ganvié côté Nokoué), S2 (Ganvié côté Sô) ; S3 (Sô-Ava) ; S4 (Sôtchanhoué) ;

$\mathrm{S} 5$ (centre du lac) et $\mathrm{S} 7$ (Embarcadère Calavi). 


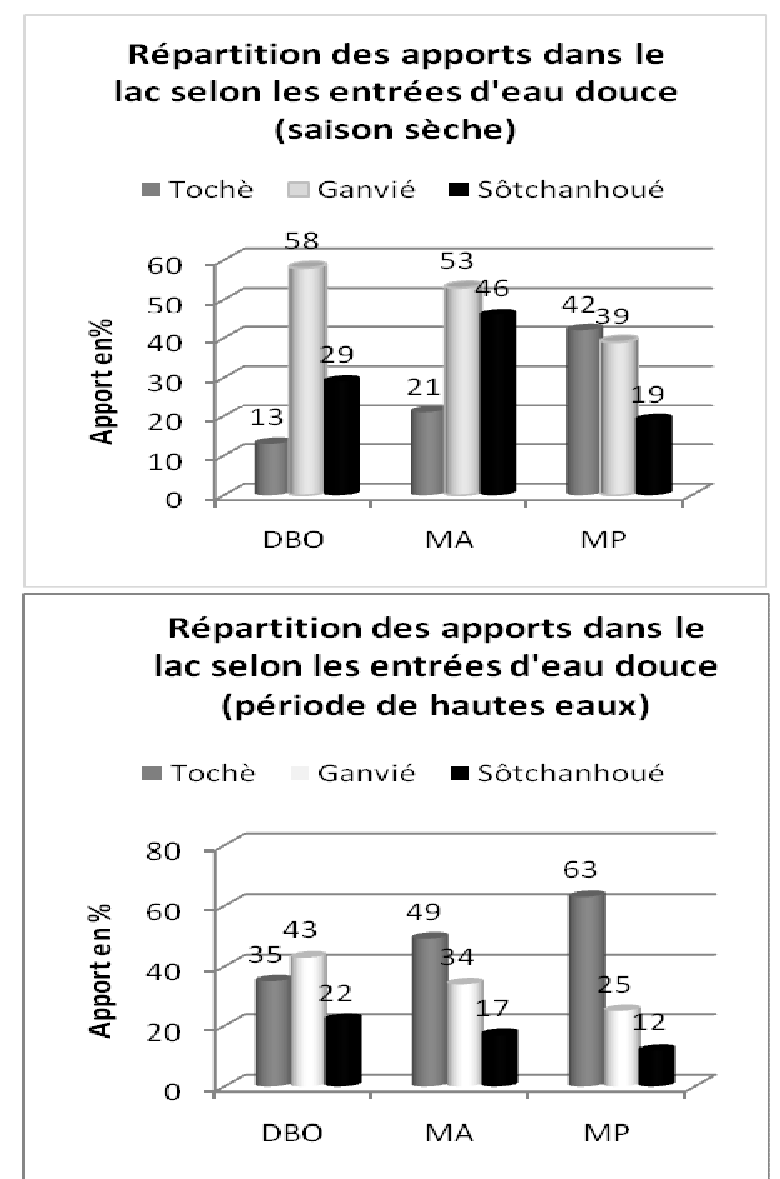

Figure 2 : Répartition des charges reçues par le lac selon les différentes entrées d'eau douce.

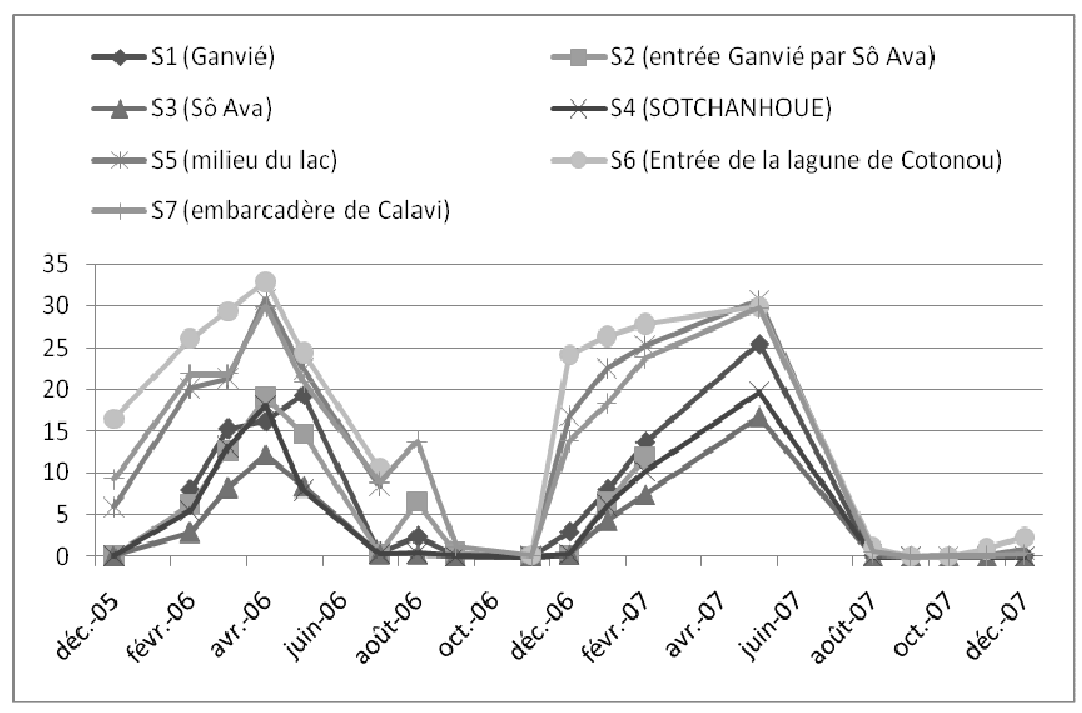

Figure 3 : Evolution saisonnière de la salinité sur le lac Nokoué. S1 (Ganvié côté Nokoué), S2 (Ganvié côté Sô) ; S3 (Sô-Ava) ; S4 (Sôtchanhoué) ; S5 (centre du lac) et S7 (Embarcadère Calavi). 


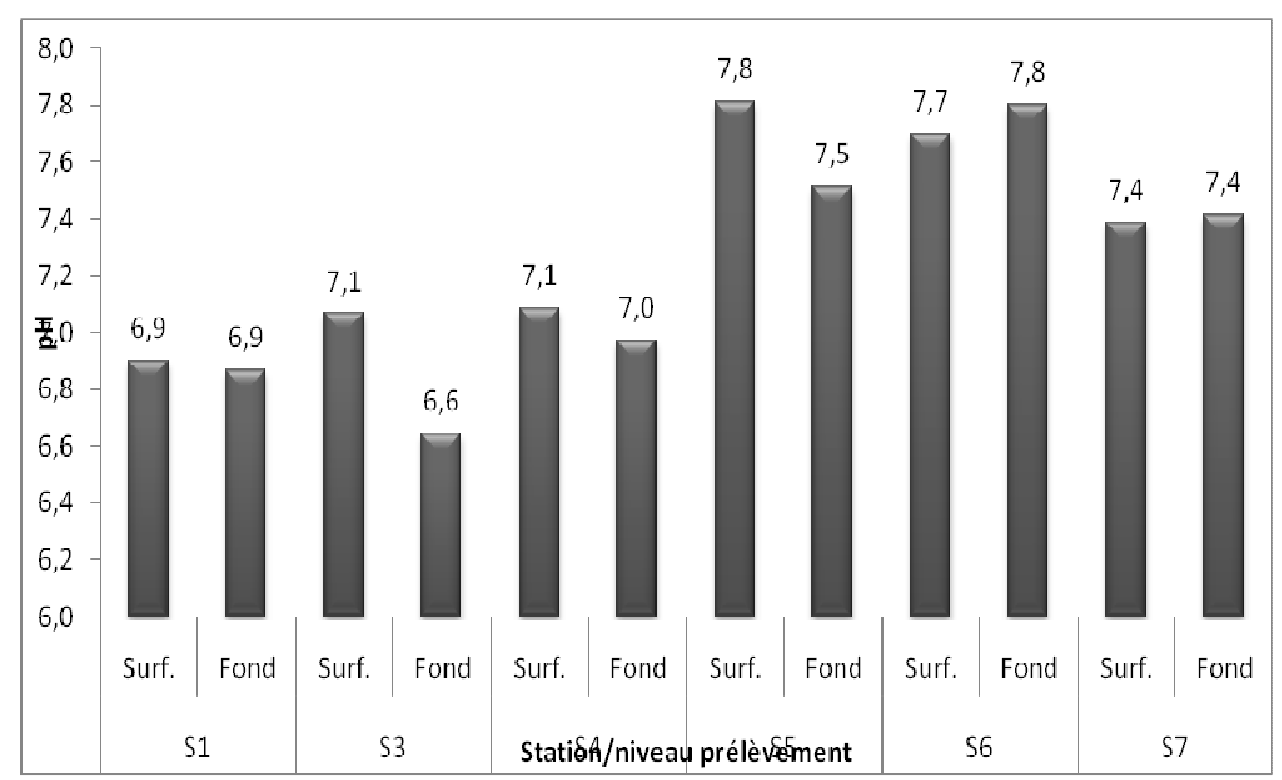

Figure 4 : Evolution comparée des valeurs moyennes du pH de l'eau de surface et de l'eau du fond du lac Nokoué en 2007.

S1 (Ganvié côté Nokoué), S2 (Ganvié côté Sô); S3 (Sô-Ava); S4 (Sôtchanhoué) ; S5 (centre du lac) et S7 (Embarcadère Calavi).

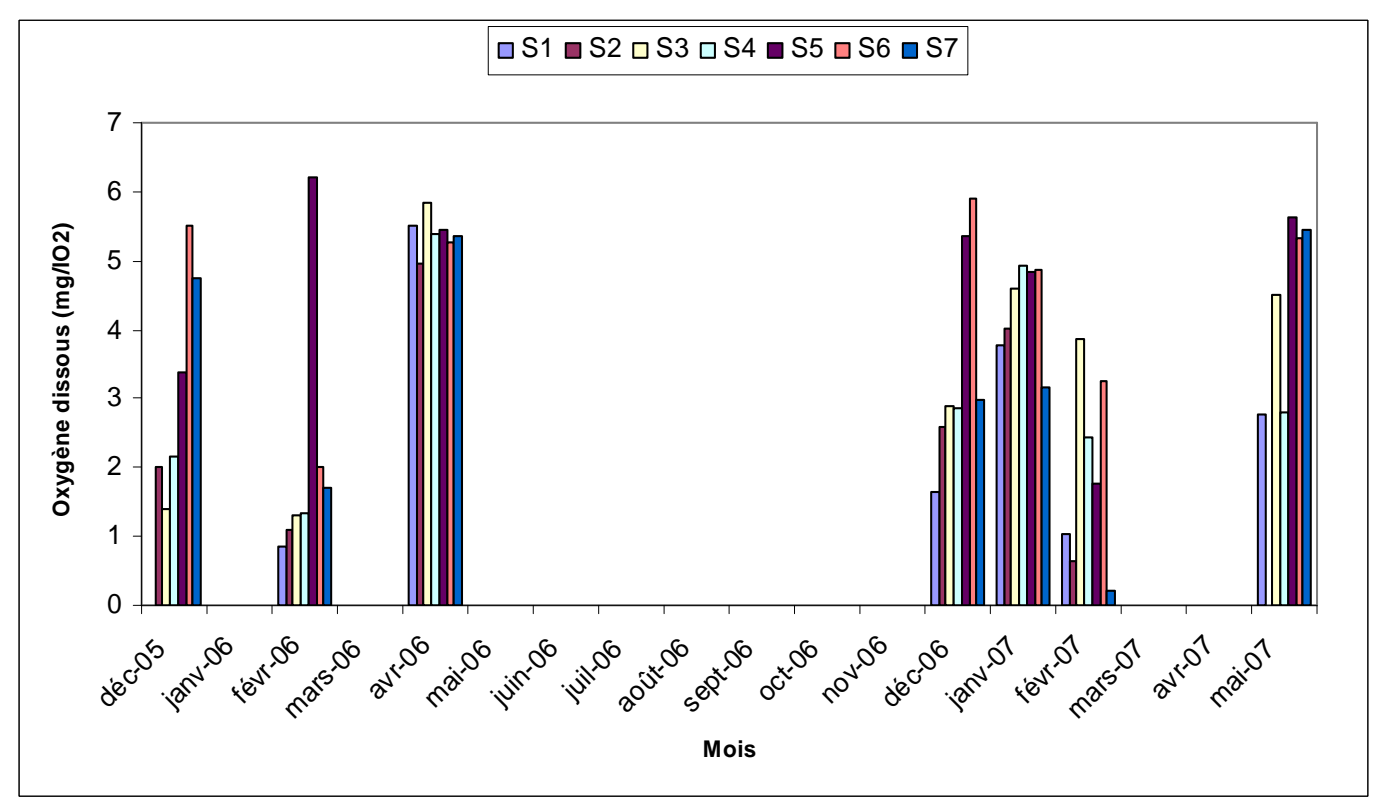

Figure 5: Evolution dans le temps de l'oxygène dissous du lac Nokoué.

S1 (Ganvié côté Nokoué), S2 (Ganvié côté Sô) ; S3 (Sô-Ava) ; S4 (Sôtchanhoué) ; S5 (centre du lac) et S7 (Embarcadère Calavi). 


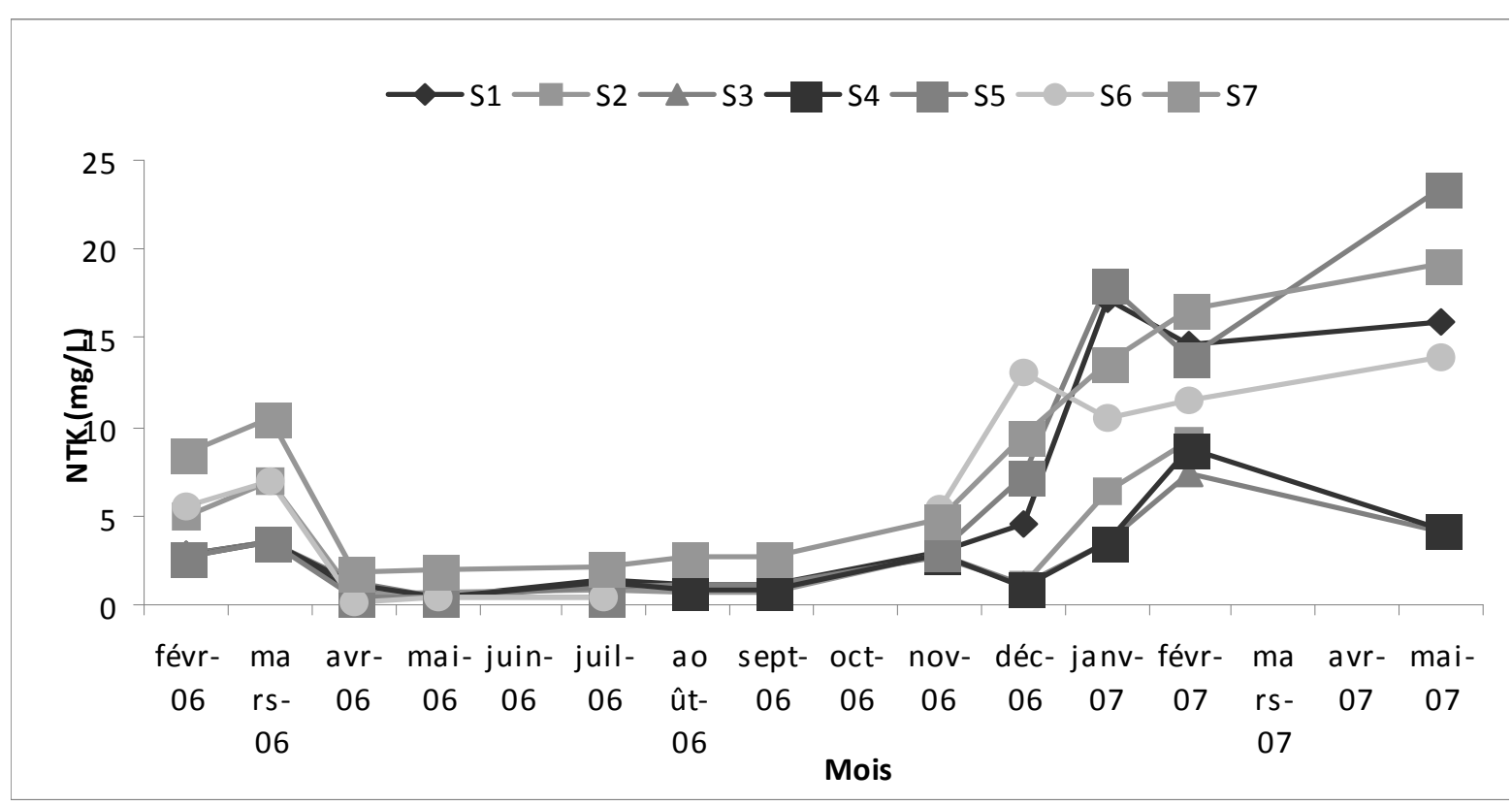

Figure 6 : Dynamique de l'évolution dans le temps de l'azote total NTK.

S1 (Ganvié côté Nokoué), S2 (Ganvié côté Sô); S3 (Sô-Ava); S4 (Sôtchanhoué) ; S5 (centre du lac) et S7 (Embarcadère Calavi). 


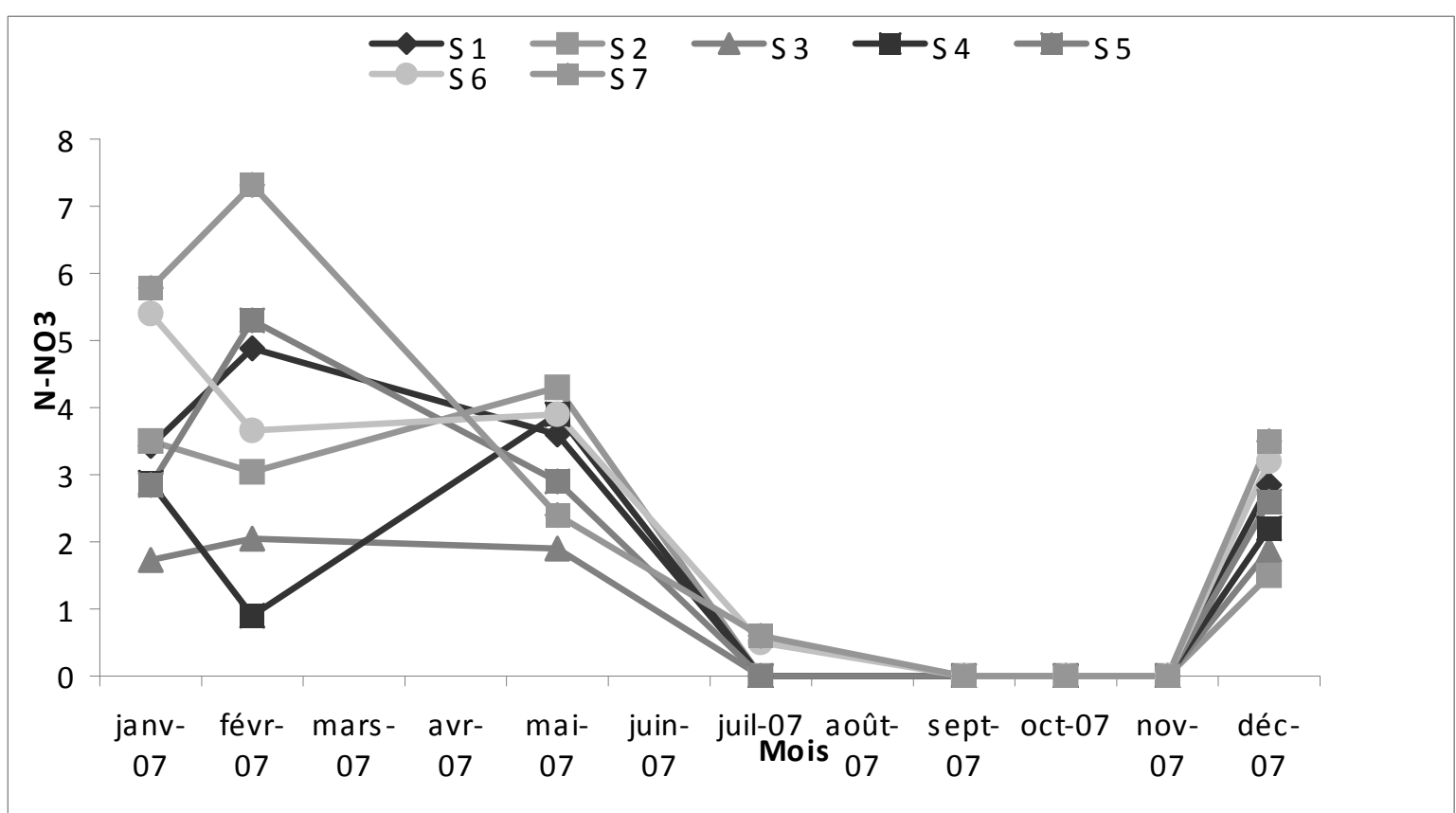

Figure 7 : Evolution des concentrations de nitrate selon les points de prélèvement du lac Nokoué S1 (Ganvié côté Nokoué), S2 (Ganvié côté Sô) ; S3 (Sô-Ava); S4 (Sôtchanhoué); S5 (centre du lac) et S7 (Embarcadère Calavi). 
D. MAMA et al. / Int. J. Biol. Chem. Sci. 5(5): 2076-2093, 2011

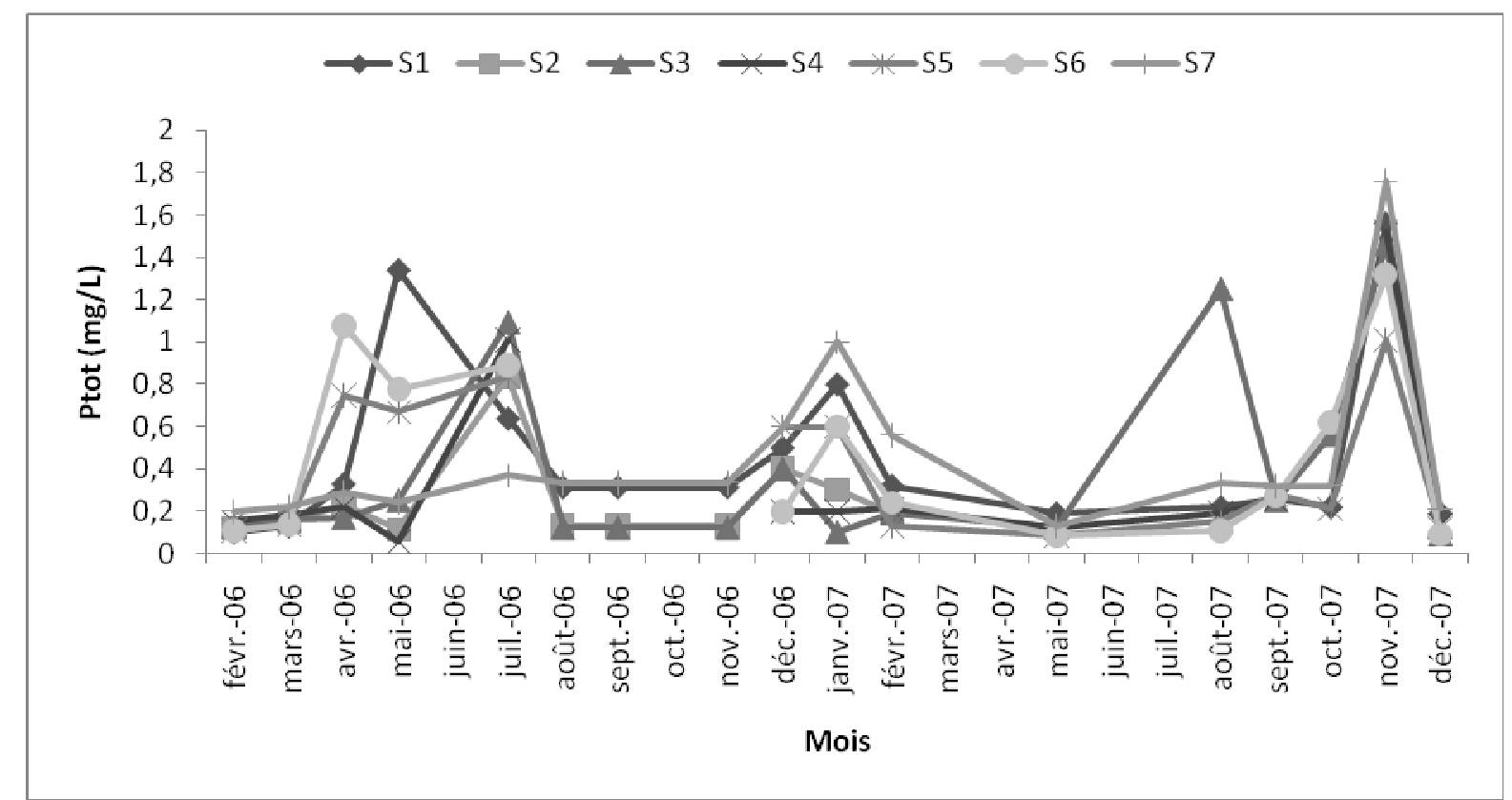

Figure 8 : Dynamique de l'évolution saisonnière du phosphore total (en haut la pluviométrie et en bas l'évolution du phosphore total. S1 (Ganvié côté Nokoué), S2 (Ganvié côté Sô) ; S3 (Sô-Ava) ; S4 (Sôtchanhoué) ; S5 (centre du lac) et S7 (Embarcadère Calavi). 
D. MAMA et al. / Int. J. Biol. Chem. Sci. 5(5): 2076-2093, 2011

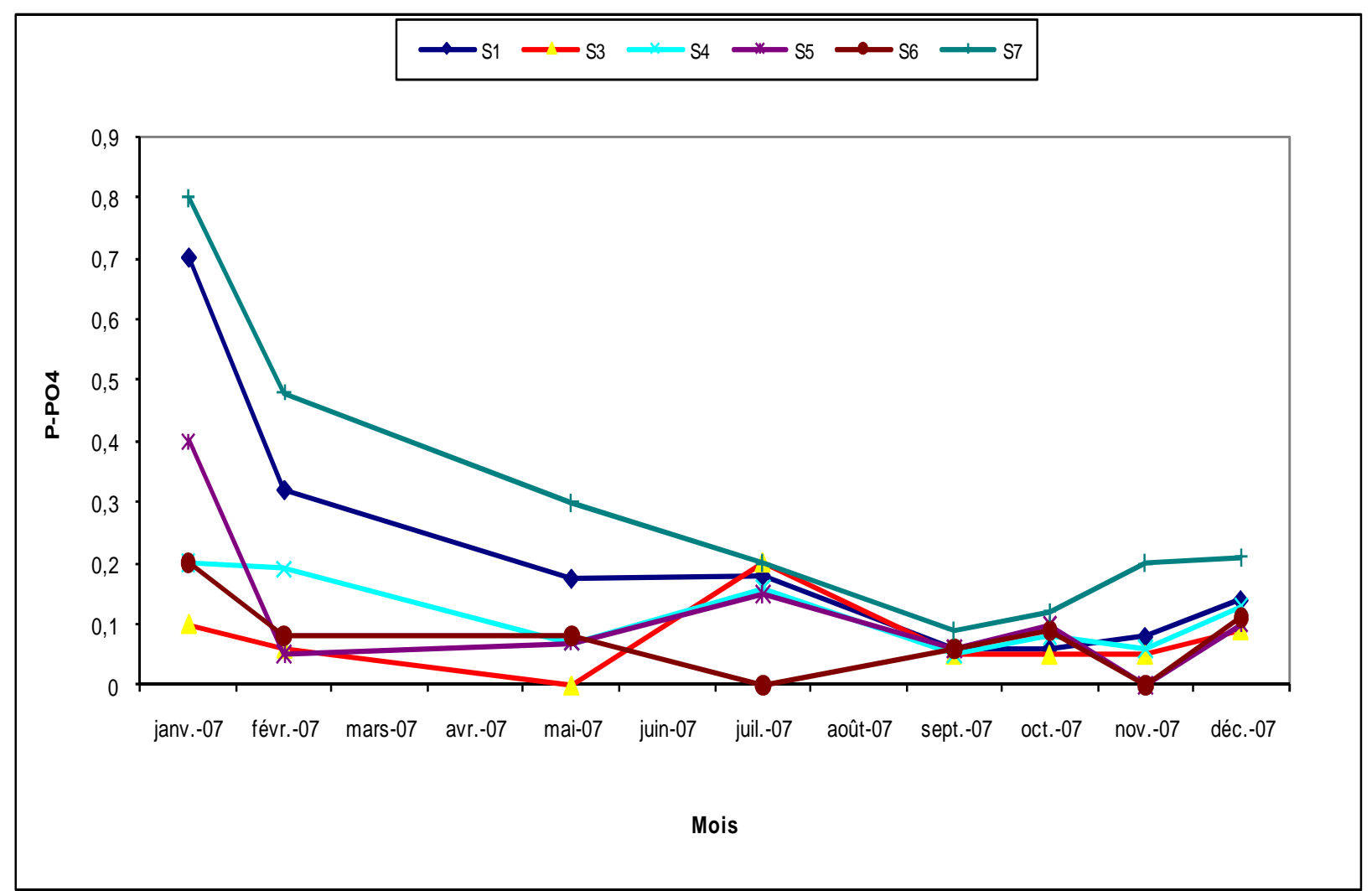

Figure 9 : Evolution des concentrations en phosphore selon les points de prélèvement du lac Nokoué. S1 (Ganvié côté Nokoué), S2 (Ganvié côté Sô) ; S3 (Sô-Ava) ; S4 (Sôtchanhoué) ; S5 (centre du lac) et S7 (Embarcadère Calavi). 


\section{DISCUSSION \\ Hydrologie}

En ce qui concerne son régime hydrologique, il faut noter qu'il est principalement contrôlé par le flux saisonnier des apports continentaux de la Sô (en saison sèche) et de l'Ouémé (en période de hautes eaux), et des échanges marins avec l'océan avec une intrusion saline surtout pendant l'étiage. Cet échange avec la mer se fait à travers :

le chenal de Totchè $(5 \mathrm{~km}$ de long et $150 \mathrm{~m}$ de large) qui rejoint la lagune de PortoNovo à l'Est.

- le chenal de Cotonou (4,5 km de long et $250 \mathrm{~m}$ de large) qui rejoint directement la mer au Sud du système et qui représente la principale voie d'échange avec l'océan.

L'influence hydrologique des tributaires sur le lac Nokoué, observée dans ce travail est en accord avec les cotes maximales IGN relevées sur la lagune de Cotonou et qui varient entre $0.93 \mathrm{~m}$ et $1.88 \mathrm{~m}$ en période de hautes eaux et entre $0,3 \mathrm{~m}$ et $0,56 \mathrm{~m}$ en saison sèche (Hounguè, 2011). Cette forte diminution du régime hydrologique en saison sèche est due à la fois à la l'absence prolongée en cette période de pluie mais également à l'évapotranspiration (Mama, 2010 ; Hounguè 2011).

Les quantités d'eau reçues en passant de la période de hautes eaux à celle de la saison sèche sont également influencées par les apparts des tributaires dans le lac subissent également une forte variation et confirment les résultats des travaux menés sur le site. En effet, selon Le Barbé et al. (1993), le volume moyen du lac est estimé à $236,4 \mathrm{M} \cdot \mathrm{m}^{3}$; en en période de crue, il est de $325,6 \mathrm{M}$. $\mathrm{m}^{3}$; à l'étiage, il n'est que de 147,1 M.m³

\section{Transport des solutés : Intrusion saline}

La salinité des eaux du lac Nokoué est maximale aux mois d'avril et de mai (période de basses eaux) et devient presque nulle en octobre et Novembre avec la dilution en période de hautes eaux. Ceci s'explique par les interactions continentales et marines dues aux apports saisonniers d'eau salée de l'océan Atlantique et d'eau douce du fleuve Ouémé et de la rivière Sô ; aussi la majeure partie de ces rives est marécageuse. Le résultat obtenu est en accord avec ceux d'autres la bibliographie qui stipule que la distribution spatiale de la salinité des eaux dans le lac Nokoué selon leur origine est sous le contrôle de l'hydrodynamique générée par les vents du lac (Boukari, 1998; Negusse, 2009).

Le régime thermique des eaux du lac favorisent le mixage et le transfert des solutés. Les eaux du lac Nokoué sont particulièrement homogènes. La température de la tranche d'eau demeure élevée sur toute l'année. Les variations journalières de la température sont beaucoup plus importantes que les variations saisonnières. Ces résultats confirment ceux de Le Barbé et al. (1993) qui fait ressortir une amplitude moyenne de $5{ }^{\circ} \mathrm{C}\left(\right.$ de $25,6{ }^{\circ} \mathrm{C}$ à $30,6{ }^{\circ} \mathrm{C}$ ) au cours de la matinée et une amplitude de $2{ }^{\circ} \mathrm{C}\left(\right.$ de $30{ }^{\circ} \mathrm{C}$ à $32^{\circ} \mathrm{C}$ ) au cours de l'après-midi.

Les caractéristiques hydrologiques ont eu un impact sur la qualité des eaux du lac à travers le transport des solutés et les effets de concentration liés à la conjugaison de l'évapotranspiration et l'intrusion saline.

\section{Qualité des eaux : charges polluantes}

Les teneurs en matière organique biodégradable (DBO) sont assez élevées, de l'ordre de $10 \mathrm{mg} / 1 \mathrm{~d}^{\prime} \mathrm{O}_{2}$. Des valeurs très élevées sont obtenues en février période forte concentration, ce qui s'explique par la décomposition des macrophytes dans le lac et l'absence de dilution par les eaux douces (fleuve Ouémé ou eaux de pluie).

La décomposition de tous ces végétaux occasionne une importante consommation d'oxygène dissous, et va s'accompagner d'une désoxygénation du milieu, en particulier à l'interface eau/sédiment.

Les sources endogènes de nutriment proviennent essentiellement du relargage à partir des sédiments. Lorsque les nutriments ont atteint un lac, ils sont recyclés entre les sédiments, les plantes aquatiques et la colonne d'eau. À terme, les nutriments devraient être 
stockés dans les sédiments. Cependant, un changement de conditions dans le lac peut entraîner le relargage des nutriments. Le relargage du phosphore vers la colonne d'eau est un processus complexe. Plusieurs mécanismes, physiques, chimiques et biologiques, interviennent. Plusieurs facteurs sont considérés comme responsables des taux de relargage du phosphore : le potentiel d'oxydoréduction, la concentration en nitrates, la minéralisation, la libération de gaz, la bioturbation, les effets du phytoplancton et des macrophytes, les caractéristiques des sédiments, un $\mathrm{pH}$ élevé et le brassage éolien (Van Hullebusch, 2002 ; Mama, 2010)

Apports exogène de nutriments dans le système: par les eaux usées rejetées directement dans le lac, les eaux de lessivage suite aux précipitations sur le bassin versant du fleuve Ouémé et de la Sô, ainsi que sur la zone urbanisée à proximité du lac.

\section{Risques d'eutrophisation}

L'évaluation du risque d'eutrophisation lac Nokoué à partir de l'outil de diagnostic d'Ifremer validé en zone tempérée sur le lac Thau en France, montre que :

Pour les sédiments, $75 \%$ des stations (S1; S2 et S7) présentent des eaux de mauvaise qualité vis-à-vis du risque d'eutrophisation en se basant sur la caractéristique des sédiments en matières organiques (MO), en azote total (NT) et en phosphore total (PT).

Au niveau de la colonne d'eau, toutes les stations sont dans un mauvais état vis-à-vis de leur état trophique.

$\mathrm{Au}$ total en prenant en compte la colonne d'eau et les sédiments, toutes les stations se classent dans un mauvais état sauf la station S5 (centre du lac) qui est classée dans la gamme «Bon ». Cette station connaît certes la présence des algues, mais pas la prolifération des jacinthes d'eau en confirmant les résultats obtenus par Mama (2010) et Negusse (2009).

La salinité du milieu est contrôlée par les apports d'eau douce du fleuve, les précipitations directes et les marées via le chenal de Cotonou. Au plan de la dynamique hydrologique, l'évolution des débits journaliers de 2000 à 2005, montre qu'au cours des mois d'août, septembre et octobre, la salinité du lac Nokoué est essentiellement influencée par le fleuve Ouémé et la rivière Sô (Mama, 2010 ; Hounguè, 2011). L'évapotranspiration, étant supérieure à la hauteur des précipitations peut contribuer significativement à la concentration des nutriments et de la salinité dans le lac. En effet, l'exploitation des données de l'ASECNA a révélé une moyenne interannuelle de l'ETP égale à 1669,7 mm à Cotonou et un taux d'évaporation durant l'année variant entre 4 et $6 \mathrm{~mm} / \mathrm{jour}$.

\section{Conclusion}

Le bilan écohydrologique montre qu'en saison sèche, les charges en DBO et en azote sont très importantes au niveau de Ganvié (58\%) et Sôtchanhoué (29\%). Pour cette saison, les apports du fleuve Ouémé sont les plus faibles. En ce qui concerne la matière phosphorée, la charge apportée par l'Ouémé à Totchè $(42 \%)$ est la plus importante. En période de hautes eaux, on assiste à un effet de dilution importante surtout pour la charge organique dont les apports semblent plus dépendre des villages lacustres et des eaux usées en provenance des quartiers environnants. Par contre, les apports des matières nutritives et surtout le phosphore semblent plus dépendre des eaux drainées à partir des bassins versants.

Selon la classification de l'OCDE, et la grille de diagnostic du risque d'eutrophisation d'Ifremer (Trinquier, 2007), les différents résultats relatifs à l'évolution saisonnière des paramètres physico chimiques, des concentrations en nutriments, montrent que le système est eutrophe pour la quasi-totalité des points suivis.

Les résultats de ce travail ont permis de disposer de données de base qui pourront être exploités pour modélisation de contrôle de la croissance des jacinthes d'eau dans le lac Nokoué. On note une évolution saisonnière du 
système. Du point de vue des facteurs de contrôle des jacinthes d'eau, il ressort que ;

la température moyenne étant de $27^{\circ} \mathrm{C}$, ce qui correspond à la température optimale de croissance des jacinthes d'eau, ce paramètre ne conditionnera donc pas le développement des jacinthes dans ce système.

la salinité inhibe totalement la croissance des jacinthes de février à juin dans l'ensemble du lac.

les nutriments contrôlent le développement des jacinthes lorsque la salinité n'inhibe plus la croissance des jacinthes, c'est-à-dire de juillet à janvier.

\section{REMERCIEMENT}

Les auteurs tiennent à remercier l'Agence Universitaire de la Francophonie (AUF) pour le soutien financier qu'elle a apporté à cette étude.

\section{REFERENCES}

Boukari M. 1998, Fonctionnement du système aquifère exploité pour l'approvisionnement en eau de la ville de Cotonou sur le littoral Béninois. Impact du développement urbain sur la qualité des ressources. Thèse d'Etat ès-sciences, UCAD de Dakar et Université Nationale du Bénin ; 277p.

Feuchtmayr H, Moran R, Hatton K, Connor L, Heyes T, Moss B, Harvay I, Atkinson D. 2009 Global warming and eutrophication: effects on water chemistry and autotrophic communities in experimental hypertrophic shalow lake mesocosms. Journal of Applied Ecology, 46: 713-723.

Hadj Amor R, Quaranta G, Gueddari F, Million D, Clauer N. 2008. The life cycle impact assessment applied to a coastal lagoon: the case of the Slimane lagoon (Tunisia) by the study of seasonal variations of aquatic eutrophication potential. Environmental Geology, 54: 1103 - 1110.

Hounguè R. 2011. Contribution à l'étude du fonctionnement hydrodynamique du lac
Nokoué. Mémoire de Licence Professionnelle en Hydrologie, Faculté des Sciences et Techniques de l'Université d'Abomey - Calavi.

Hill PM, Coetzee JA. 2008 Integrated control of water hyacinth in Africa. EPPO Bulletin, 38 : 452 - 457.

Le Barbé L, Alé G, Millet B, Texier H, Borel Y, Gualde TR. 1993. Les Ressources en Eaux Superficielles de la République du Bénin. ORSTOM ; 540p.

Mama D. 2010. Méthodologie et résultats du diagnostic de l'eutrophisation du lac Nokoué (Benin). Thèse de doctorat, Université de Limoges ; 157p.

Ogutu -Ohwayo R, Hecky RE, Cohen SA, Kauf L. 1997. Human Impacts on the African Great Lakes. Environmental Biology of Fishes, 50: 117-137.

Ryding SO, Rast W. 1994. Contrôle de l'Eutrophisation des Lacs et des Réservoirs. Collection des Sciences de l'Environnement $\mathrm{n}^{\circ}$ 9. Masson éditeur : Paris ; 294 p.

Trinquier Christel. 2009. Le risque d'eutrophisation des lagunes méditerranéennes: le cas de la lagune de Thau. Mémoire de Master 1, Université Paul Valéry - Monpellier III ;

Van Hullebusch, E, Deluchat V, Chazal PM, Baudu M. 2002b. Environmental impact of two successive chemical treatments in a small shallow eutrophied lake: (II) case of copper sulfate. Environmental Pollution, 120: 627-634.

Wilson JR, Rees M, Thomas M, Hill G. 2001. Water hyacinth population dynamics. ACIAR Proceedings 102, pp. 96-104.

Wilson JR, Holst N, Rees M. 2005. Determinants and patterns of population growth in water hyacinth. Aquatic Botany, 81: 51-67. 